\title{
ON THE ZEROS OF A CLASS OF GENERALISED DIRICHLET SERIES-VII
}

\author{
K. Ramachandra
}

\begin{abstract}
Let $a_{1}=1=\lambda_{1}$ and $\left\{\lambda_{n}\right\}(n=1,2,3, \ldots)$ a sequence of real numbers with $1 / c \leq \lambda_{n+1}-\lambda_{n} \leq c(c \geq 1$ is a constant $)$. Let $\left\{a_{n}\right\}(n=1,2,3, \ldots)$ be any sequence of complex numbers satisfying

$$
x^{A} \geq \frac{1}{x} \sum_{n \leq x}\left|a_{n}\right|^{2} \geq d_{1} \exp \left(-\frac{d_{2} \log x}{\log \log x}\right)
$$
\end{abstract}

for all $x \geq x_{0}(A)$ where $A, d_{1}, d_{2}$ are positive constants. Suppose that $F(s)=\sum_{n=1}^{\infty}\left(a_{n} \lambda_{n}^{-s}\right)$ $(\sigma>A+10)$ can be continued analytically in $\left(\sigma \geq \frac{1}{2}-\delta, T \leq t \leq 2 T\right)$ and there $\max |F(s)| \leq T^{A}$, where $A$ and $\delta$ are positive constants with $0<\delta<\frac{1}{2}$ ( $A$ may be the same as before). Let $0<\varepsilon<1$ and $D=D(\varepsilon)=D\left(\varepsilon, c, d_{1}, d_{2}, A, \delta\right)$ a suitable constant depending on the parameters indicated. Then it is proved that $F(s)$ has at least $T^{1-\varepsilon}$ zeros in the rectangle

$$
\left\{\sigma \geq \frac{1}{2}-\frac{D}{\log \log T}, T \leq t \leq 2 T\right\}
$$

provided $T$ exceeds a large constant.

\section{Introduction}

Let $s=\sigma+i t, \sigma>0$ and

$$
\zeta(s)=\sum_{n=1}^{\infty}\left(n^{-s}-\int_{n}^{n+1} u^{-s} d u\right)+\frac{1}{s-1} .
$$

It is well-known that $\zeta(s)$ has infinity of zeros with $\sigma=\frac{1}{2}$. (Here ample use is made of the functional equation for $\zeta(s))$. But a proof that $\zeta(s)$ has infinity of zeros in $\sigma \geq \frac{1}{2}$, a proof which does not use the functional equation is not known. As such a good generalisation of this fact to Dirichlet series $F(s)$ more general than $\zeta(s)$ is not known. For example consider $F_{0}(s)=\sum_{n=1}^{\infty}\left((-1)^{n} \lambda_{n}^{-s}\right)$ where $\lambda_{1}=1$ and $1 / c \leq \lambda_{n+1}-\lambda_{n} \leq c$ where $c \geq 1$ is a constant. This is clearly analytic in $\sigma>0$ and we do not know how to prove that $F_{0}(s)$ has infinity of zeros in $\sigma \geq \frac{1}{2}$. With these problems in view R. Balasubramanian and K. Ramachandra undertook some investigations in the earlier papers of this series. An example of some elegance discovered by them is the following theorem (for more general results see the earlier papers of the series viz. $[R]_{1},[R]_{2},[\mathrm{BR}]_{2},[\mathrm{BR}]_{3},[\mathrm{R}]_{3}$ and $\left.[\mathrm{BR}]_{4}\right)$.

AMS 1991 Subject Classification: 11XX, 11MXX, 11M41. 
Theorem 1. Let $\chi(n)(n=1,2,3, \ldots)$ be any sequence of complex numbers with $\sum_{n \leq x} \chi(n)=O(1)$. Then the number of zeros of $\zeta(s)+\sum_{n=1}^{\infty} \chi(n) n^{-s}$ in the rectangle $\left(\left|\sigma-\frac{1}{2}\right| \leq \delta, T \leq t \leq 2 T\right)$ ( $\delta$ is an arbitrary constant subject to $0<\delta<\frac{1}{2}$ is

$$
\gg T \log T \text {. }
$$

In this paper I consider the series

$$
F(s)=\sum_{n=1}^{\infty} a_{n} \lambda_{n}^{-s}
$$

where $a_{1}=1, \lambda_{1}=1,1 / c \leq \lambda_{n+1}-\lambda_{n} \leq c(c \geq 1$ is a constant $)$ and for all $x \geq e^{e}$,

$$
\frac{1}{x} \sum_{n \leq x}\left|a_{n}\right|^{2} \geq d_{1} \operatorname{Exp}\left(-\frac{d_{2} \log x}{\log \log x}\right)
$$

$d_{1}>0, d_{2}>0$ being positive constants. Further I assume that $\left|a_{n}\right| \leq n^{A}$ and that $F(s)$ has analytic continuation in $\left(\sigma \geq \frac{1}{2}-\delta, T \leq t \leq 2 T\right)$ and here $|F(s)| \leq T^{A}$ where $A$ and $\delta$ are positive constants (with $0<\delta<\frac{1}{2}$ ) and $T \geq T_{0}(A, \delta)$. I prove the following theorem.

Theorem 2. Let $0<\varepsilon<1$ and $D=D(\varepsilon)=D\left(\varepsilon, c, d_{1}, d_{2}, A, \delta\right)$ a suitable constant depending on the parameters indicated. Let $T \geq T_{0}=$ $T_{0}\left(\varepsilon, c, d_{1}, d_{2}, A, \delta\right)$. Then $F(s)$ has at least $T^{1-\varepsilon}$ zeros in the rectangle

$$
\left\{\sigma \geq \frac{1}{2}-\frac{D}{\log \log T}, T \leq t \leq 2 T\right\}
$$

In particular this theorem is true for the function $F_{0}(s)$ stated in the introduction. It is also true of the function of Theorem 1.

\section{Proof of Theorem 2.}

The proof can be broken up conveniently into a few lemmas. I assume that $T \geq T_{0}$ (a constant which may depend on other constants). I need the condition (4) only from Lemma 10 onwards. We write $T_{1}$ for any number between $T+H$ and $2 T-H$.

Lemma 1. Let $\alpha>\delta$ and $R=R(H, \alpha)$ denote the rectangle $\left(\sigma \geq \alpha, T_{1}-\right.$ $\left.H \leq t \leq T_{1}+H\right)$. Let $F(s)$ be as above and continuable analytically in $R(H, \alpha-\delta)$ 
and here $|F(s)| \leq T^{A}$. Further let $F(s) \neq 0$ in $R(H, \alpha)$. Then for $t=T_{1}$ in $R(H, \alpha)$ we have

(6) $-A_{1} \frac{\log T}{\log \log T} \max \left[1, \log \left\{\frac{A_{2}}{(\sigma-\alpha) \log \log T}\right\}\right] \leq \log |F(s)| \leq A_{3} \frac{\log T}{\log \log T}$,

and

$$
|\arg F(s)| \leq A_{4} \frac{\log T}{\log \log T},
$$

provided $\frac{1}{2} T \geq H \geq A_{5} \log \log \log T$. (Here $A_{1}, A_{2}, A_{3}, A_{4}$, and $A_{5}$ are suitable constants depending on $A, \delta$ and $c$. Also $\alpha$ can depend on $T$ and $H$ ).

Proof. See the appendix in [RS].

Lemma 2. In $\left(\sigma \geq \alpha+(\log \log T)^{-1}, T_{1}-H \leq t \leq T_{1}+H\right)$ we have

$$
|\log F(s)| \leq A_{6} \frac{\log T}{\log \log T},
$$

provided $t=T_{1}$ and $H \geq A_{5} \log \log \log T$.

Proof. Follows from Lemma 1.

Lemma 3. Let $z=x+i y$ be a complex variable subject to $|x| \leq \frac{1}{4}$. Then

$$
\left|\operatorname{Exp}\left((\sin z)^{2}\right)\right| \leq \begin{cases}2 & \text { for all } y \\ 2(\operatorname{Exp} \operatorname{Exp}|y|)^{-1} & \text { for }|y| \geq 2 .\end{cases}
$$

Remark. This important function viz. $\operatorname{Exp}\left((\sin z)^{2}\right)$ was introduced and used extensively by me in my earlier papers.

Proof. The proof is really simple. See for example Lemma 2.1 of $[\mathrm{BR}]_{1}$ for a proof.

Lemma 4. Let $0<\delta<\frac{1}{4}, H \geq A_{7} \log \log T, \alpha_{1}=\alpha-\delta, \alpha_{2}=\alpha-$ $(10 D) /(\log \log T), \alpha_{3}=\alpha$, where $A_{7}$ is a large positive constant. Then for $T_{1}-\frac{1}{2} H \leq t \leq T_{1}+\frac{1}{2} H$ we have uniformly,

$$
\left|F\left(\alpha_{2}+i t\right)\right| \leq 1000\left(T^{A}\right)^{\mu}\left(\operatorname{Exp}\left(A_{3} \frac{\log T}{\log \log T}\right)\right)^{1-\mu},
$$

(where $\mu=10 D \delta^{-1}(\log \log T)^{-1}$ ) and so, by maximum modulus principie, the same holds uniformly for $\alpha_{2}$ replaced by any $\sigma \geq \alpha-(10 D) /(\log \log T)$ and $T_{1}-\frac{1}{3} H \leq t \leq T_{1}+\frac{1}{3} H$. 
Proof. Put $s_{0}=\alpha_{2}+i t$ and (for a complex variable $w$ ),

$$
\varphi(w)=X^{w-s_{0}} F(w) \operatorname{Exp}\left(\sin ^{2}\left(w-s_{0}\right)\right),
$$

$X$ being a positive parameter. Apply maximum modulus principle to the rectangle

$$
T_{1}-\frac{3}{4} H \leq \operatorname{Im} w \leq T_{1}+\frac{3}{4} H, \alpha_{1} \leq \operatorname{Re} w \leq \alpha_{3} .
$$

We have

$$
\begin{aligned}
\left|\varphi\left(s_{0}\right)\right|= & \left|F\left(s_{0}\right)\right| \\
\leq & 2\left\{X^{10 D / \log \log T}\left(X^{-\delta} T^{A}+\operatorname{Exp}\left(\frac{A_{3} \log T}{\log \log T}\right)\right)\right\} \\
& +2 T^{A}\left(X^{\delta}+X^{-\delta}\right) \cdot 2\left(\operatorname{Exp} \operatorname{Exp} \frac{H}{4}\right)^{-1} .
\end{aligned}
$$

Choosing $X$ by $X^{\delta}=T^{A} \operatorname{Exp}\left(\left(-A_{3} \log T\right) /(\log \log T)\right)$ we obtain the first result. Since $F(s)=O(1)$ when the real part of $s$ is large and also $|F(s)| \leq T^{A}$ (in the relevant rectangle) the second result follows from the first by a similar application of the maximum modulus principle. We have to use the function $\operatorname{Exp}\left((\sin (z / B))^{2}\right)$ (where $B$ is a large constant) in the proof.

Lemma 5. (Borel-Caratheodory Theorem). Suppose $f(z)$ is analytic in $\left|z-z_{0}\right| \leq R$ and on $\left|z-z_{0}\right|=R$ we have $\operatorname{Re} f(z) \leq U$. Then in $\left|z-z_{0}\right| \leq r<R$, we have,

$$
|f(z)| \leq \frac{2 r U}{R-r}+\frac{R+r}{R-r}\left|f\left(z_{0}\right)\right| .
$$

Proof. See page 174 of $[\mathrm{T}]$.

Lemma 6. Let $\alpha_{4} \geq \alpha+(\log \log T)^{-1}, s_{0}=\alpha_{4}+i t, T_{1}-\frac{1}{4} H \leq t \leq T_{1}+\frac{1}{4} H$. Consider circles of radius $R=11 D(\log \log T)^{-1}$ and $r=10 D(\log \log T)^{-1}$ both with centre $s_{0}$. Then in $\left(\sigma \geq \alpha-9 D(\log \log T)^{-1}, T_{1}-\frac{1}{4} H \leq t \leq T_{1}+\frac{1}{4} H\right)$ we have

$$
|\log F(s)|=O\left(D \log T(\log \log T)^{-1}\right),
$$

provided that in $\left(\sigma \geq \alpha-10 D(\log \log T)^{-1}, T_{1}-H \leq t \leq T_{1}+H\right)$ we have $F(s) \neq 0$.

Proof. Follows from the fact that both $r(R-r)^{-1}$ and $(R+r)(R-r)^{-1}$ are $O(1)$. We take $f(z)$ to be $\log F(z)$. This proves the lemma. (Of course we have written $z$ for $s$ for the purposes of application of Borel-Caratheodory's theorem in the notation of Lemma 5. But this should not cause confusion.) 
Lemma 7. Let $A_{8}>0$ be an arbitrary constant which is fixed independent of D. Put $\sigma_{2}=\alpha-A_{8}(\log \log T)^{-1}$. Then in $\left(\sigma=\sigma_{2}, T_{1}-(1 / 5) H \leq t \leq\right.$ $\left.T_{1}+(1 / 5) H\right)$ and so in $\left(\sigma \geq \sigma_{2}, T_{1}-(1 / 6) H \leq t \leq T_{1}+(1 / 6) H\right)$, we have,

$$
\log F(\sigma+i t)=O\left(D e^{-D \lambda} \log T(\log \log T)^{-1}\right),
$$

where $\lambda>0$ is a constant independent of $D$, provided $F(s) \neq 0$ in $(\sigma \geq \alpha-$ $\left.(10 D) /(\log \log T), T_{1}-H \leq t \leq T_{1}+H\right)$.

Proof. We apply convexity argument to the three lines $\sigma=\sigma_{1}=\alpha-$ $9 D(\log \log T)^{-1}, \sigma=\sigma_{2}=\alpha-A_{8}(\log \log T)^{-1}$ and $\sigma=\sigma_{3}=\beta$ (where $\beta>0$ is such that for $\sigma \geq \beta$ we have $|\log F(s)| \leq 2$ ). Let $s_{0}=\sigma_{2}+i$ (with $\left.T_{1}-(1 / 5) H \leq t \leq T_{1}+(1 / 5) H\right)$ and

$$
\psi(w)=X^{w-s_{0}}(\log F(w)) \operatorname{Exp}\left(\sin ^{2}\left(\frac{w-s_{0}}{B}\right)\right)
$$

where $B>0$ is a large constant. Choose the rectangle $\left(\sigma_{1} \leq \sigma \leq \sigma_{3}, T_{1}-\frac{1}{4} H \leq\right.$ $\left.t \leq T_{1}+\frac{1}{4} H\right)$. We get as in the proof of Lemma 4

$$
\left|\log F\left(s_{0}\right)\right| \leq C_{1}\left(\frac{C_{2} D \log T}{\log \log T}\right)^{\theta_{1}} 2^{\theta_{2}}
$$

where $C_{1}$ and $C_{2}$ are positive constants independent of $D$ and

$$
\theta_{1}=\frac{\sigma_{3}-\sigma_{2}}{\sigma_{3}-\sigma_{1}} \quad \text { and } \quad \theta_{2}=\frac{\sigma_{2}-\sigma_{1}}{\sigma_{3}-\sigma_{1}} .
$$

Since $\theta_{1}<1$ and $\theta_{2}<1$ we have

$$
\left|\log F\left(s_{0}\right)\right| \leq C_{3} D\left(\frac{\log T}{\log \log T}\right)^{\theta_{1}}
$$

where $\theta_{1} \leq 1-C_{4}(D+O(1))(\log \log T)^{-1}$. Thus the first part of the lemma is proved. The second part follows by a similar application of the maximum modulus principle.

Collecting, we have,

Lemma 8. Let $F(s) \neq 0$ in $\left(\sigma \geq \alpha-(10 D) /(\log \log T), T_{1}-H \leq t \leq\right.$ $\left.T_{1}+H\right)$ and let $A_{8}$ be any positive constant independent of $D$. Then, we have, in $\left(\sigma \geq \sigma_{2}=\alpha-A_{8}(\log \log T)^{-1}, T_{1}-H / 6 \leq t \leq T_{1}+H / 6\right)$ the inequality

$$
|\log F(s)| \leq \frac{\varepsilon^{2} \log T}{\log \log T}
$$

where $\varepsilon$ is an arbitrary constant subject to $0<\varepsilon<1$, provided $D=D(\varepsilon)$. 
Lemma 9. Let $H=T^{\varepsilon}$ and $\alpha=\frac{1}{2}$. Then for $\sigma=\sigma_{2}$, we have,

$$
\frac{1}{H} \int_{T_{1}-H / 6}^{T_{1}-H / 6}|F(s)|^{2} d t \geq c_{5} \sum_{n \leq c_{6} H}\left|a_{n}\right|^{2} n^{-2 \sigma} .
$$

Proof. It is possible to prove what we want i.e. a result weaker than (22) in a simpler way but in order to reduce the length of the present paper we refer the reader to the third main theorem in $[\mathrm{BR}]_{1}$.

Lemma 10. Choose $A_{8}=2 d_{2}$ then

$$
\max _{\left|t-T_{1}\right| \leq H / 6}\left|F\left(\sigma_{2}+i t\right)\right| \gg \operatorname{Exp}\left(d_{2} \varepsilon \frac{\log T}{\log \log T}\right) .
$$

Proof. Trivially by the condition (4), the square root of the right hand side of $(22)$ is

$$
\gg \operatorname{Exp}\left(\frac{A_{8} \log H}{\log \log T}-\frac{1}{2} d_{2} \frac{\log H}{\log \log H}\right) \gg \operatorname{Exp}\left(d_{2} \frac{\log H}{\log \log T}\right) \gg \operatorname{Exp}\left(d_{2} \varepsilon \frac{\log T}{\log \log T}\right) .
$$

The lemma is completely proved.

Now (21) and (23) contradict each other if $\varepsilon$ is a small positive constant. This proves that if $H=T^{\varepsilon}$ every rectangle $\left(\sigma \geq \frac{1}{2}-10 D /(\log \log T),\left|t-T_{1}\right| \leq H\right)$ contains a zero of $F(s)$. This proves Theorem 2 completely.

Added in proof. (i) R. Balasubramanian and myself have proved some general results regarding the zeros of a class of generalised Dirichlet series in the rectangle $\left(\sigma \geq \frac{1}{2}-C_{0}(\log \log T)^{3 / 2}(\log T)^{-1 / 2}, T \leq t \leq 2 T\right)$ where $C_{0}$ is a large positive constant depending on the Dirichlet series. The results are not too general; but they cover functions like those referred to in Theorem 1. The lower bound for the number of zeros is $\gg T(\log \log T)^{-1}$.

(ii) We have also proved (using the expression (1) for $\zeta(s)$ and also the Euler product for $\zeta(s))$ that $\zeta(s)$ has $\gg T(\log \log \log T)^{-1}$ zeros in $\left(\left|\sigma-\frac{1}{2}\right| \leq\right.$ $\left.C_{0}(\log \log T)(\log T)^{-1}, T \leq t \leq 2 T\right)$.

For the results (i) and (ii) see our papers VIII and IX with the same title as the present one in pages 21-33 and 33-43 of Hardy-Ramanujan Journal 14, 1991.

\section{References}

$[B R]_{1}$ Balasubramanian, R., and K. Ramachandra: Proof of some conjectures on the meanvalue of Titchmarsh series-I. - Hardy-Ramanujan J. 13, 1990, 1-20.

$[\mathrm{BR}]_{2}$ Balasubramanian, R., and K. Ramachandra: On the zeros of a class of generalised Dirichlet series-III. - J. Indian Math. Soc. 41, 1977, 301-315.

$[\mathrm{BR}]_{3}$ Balasubramanian, R., and K. Ramachandra: On the zeros of a class of generalised Dirichlet series-IV. - J. Indian Math. Soc. 42, 1978, 135-142. 
[BR $]_{4}$ Balasubramanian, R., and K. Ramachandra: On the zeros of a class of generalised Dirichlet series-VI. - Ark. Mat. 19, 1981, 239-250.

$[R]_{1}$ Ramachandra, K.: On the zeros of a class of generalised Dirichlet series-I. - J. Reine Angew. Math. 273, 1975, 31-40, Addendum 60.

$[\mathrm{R}]_{2}$ RAmachandRa, K.: On the zeros of a class of generalised Dirichlet series-II. - J. Reine Angew. Math. 289, 1977, 174-180.

$[R]_{3}$ Ramachandra, K.: On the zeros of a class of generalised Dirichlet series-V. - J. Reine Angew. Math. 303/304, 1978, 295-313.

[RS] Ramachandra, K., and A. Sankaranarayanan: On some theorems of Littlewood and Selberg-III (Appendix therein). - To appear.

[T] Titchmarsh, E.C.: The theory of functions. - Oxford University Press, Oxford, 1939.

Tata Institute of Fundamental Research

School of Mathematics

Homi Bhabha Road

Colaba

Bombay 400005

India

Received 4 February 1991 